\title{
Late onset ornithine transcarbamylase deficiency in a 61 year old male
}

\author{
Thomas Malfait ${ }^{1}$, Gert De Schoenmakere ${ }^{2}$, Filip Gallant ${ }^{3}$, Hans Schepkens ${ }^{2}$, Ann Van Loo ${ }^{4}$, Wouter \\ Meersseman $^{5}$, David Cassiman ${ }^{5}$, Steven Brabant ${ }^{3}$, Johannes Häberle ${ }^{6}$, Wim Terryn ${ }^{1,4}$ \\ 1. Department of Internal Medicine, Ghent University Hospital, Ghent, Belgium. 2. Department of Internal Medicine, H \\ Hart Hospital, Roeselare, Belgium. 3. Department of Anesthesiology, H Hart Hospital, Roeselare, Belgium. 4. Department \\ of Internal Medicine, Jan Yperman Hospital, Ieper, Belgium. 5. Metabolic Center, University Hospital Leuven, Leuven, \\ Belgium. 6. Department of Metabolism, University Children's Hospital, Zurich, Switzerland.
}

Correspondence: Gert De Schoenmakere. Address: Department of Nephrology, H Hart Hospital Roeselare, Wilgenstraat 2,8800 Roeselare - Belgium. Email: gdeschoenmakere@hhr.be

Received: November 25, 2014

DOI : $10.5430 /$ crim.v2n2p26
Accepted: December 21, 2014 Online Published: March 1, 2015

URL: http://dx.doi.org/10.5430/crim.v2n2p26

\section{Abstract}

Ornithine transcarbamylase (OTC) deficiency is the most frequent innate urea cycle disorder (UCD) and is the only one with an X-linked inheritance. OTC deficiency leads to hyperammonemia and subsequent elevated cerebral pressure and brain damage. Most often, UCDs are described as acute onset hyperammonemia in the newborn; however, rare, it can present in adulthood. We present the case of a 61-year old Caucasian man with unexplained coma. He developed an acute hyperammonemic encephalopathy leading to mental problems, seizures and coma. Despite the classical treatment with hemodialysis and the administration of a combination of sodium phenylacetate and sodium benzoate, the patient unfortunately died of cerebral herniation. Postmortem, genetic analysis confirmed the presence of a c.662G $>$ A (p.Ala208Thr) pathogenic mutation in the OTC gene in a hemizygous state. Synopsis: The association of neuropsychiatric symptoms or encephalopathy of unknown origin should lead, even in the absence of liver disease and even in late adulthood, to a high level of suspicion of hyperammonemia secondary to UCDs to ensure a timely diagnosis and life-saving treatment. UCD, an inborn error of metabolism, can present itself at an advanced age; attenuated phenotypes probably remain under-ascertained.

\section{Keywords}

Ornithine transcarbamylase deficiency, Hyperammonemia, Urea cycle disorder

\section{I ntroduction}

The urea cycle is the metabolic pathway that converts nitrogen, mostly in the form of ammonium (NH4+), from peripheral (muscles) and enteral sources (protein ingestion) into urea that is water soluble and can be renally excreted ${ }^{\text {[1] }}$. Urea production out of almost all of the ammonia occurs mainly in the liver preventing the accumulation of ammonia in cells and biological fluids ${ }^{[1]}$.

Urea cycle disorders represent one of the most common groups of inborn errors of metabolism and are caused by a partial or complete inactivity of one of the six enzymes, the two substrate transporters or cofactors in the pathway ${ }^{[1-3]}$. Of these, 
ornithine transcarbamoylase (OTC) deficiency is the most frequent innate, enzymatic deficiency of the urea cycle with an estimated prevalence of 1:8.000 to 1:45.000 and is the only one transmitted as an X-linked trait. Clinically, the effects of the deficiency are caused by the toxic accumulation of ammonia in the brain, which include lethargy, vomiting, behavioral and neurological abnormalities but can cause in severe cases coma, brain edema and death (cerebral herniation) ${ }^{[1-3]}$.

We present the case of fatal hyperammonemia in a 61-year old Caucasion man with ornithine transcarbamoylase deficiency. The patient developed mental problems, seizures, coma and he eventually died of cerebral herniation.

We conclude that urea cycle defects, as inborn errors of metabolism, can be clinically apparent at adult age and, if not recognised, can be fatal. The association of neuropsychiatric findings or unexplained or nonresponsive, with hyperammonemia, normal liver and kidney function needs a timely diagnosis and life-saving treatment of a urea cycle disorder.

\section{Case report}

A 61 year old Caucasian man was admitted to the psychiatric emergency ward because of acute onset of confusion and psychotic behavior. The months preceding this hospitalization he had developed progressive symptoms of anhedonia (exhaustion, loss of appetite), allegedly attributed to a theft in his shop a few months earlier. The general practitioner treated this with ethylloflazepate $2 \mathrm{mg}$ (Sanofi Aventis) and lormetazepam $1 \mathrm{mg}$ (Pfizer). Of note, his relatives mentioned a comparable episode of psychic distress 20 years before, at that time without clear diagnosis. His general practitioner and family refer patient now because of impaired speech since less than an hour; he was also seen talking to himself and he had urinated against a wall.

On day one at admission clinical evaluation was normal. Neurological status only revealed a slurred, inconsistent speech; Glasgow Coma Scale was 13/15. Standard blood tests taken in the emergency ward were normal (liver tests, ionogram, kidney function, infectious parameters, hematology, anion gap); urine sediment was normal, as was toxicology, but for the presence of benzodiazepine. A brain CT scan was normal.

The patient was tentatively diagnosed with depression and was admitted to the psychiatry ward where sulpiride $50 \mathrm{mg}$ twice-daily and prazepam $5 \mathrm{mg}$ three-times-daily were initiated. The next day no difference in neurological status was seen; patient still suffered from a slurred, inconsistent speech. On day 3 after admission, his neurological status however deteriorated rapidly, resulting in generalized seizures and coma. Electroencephalography was suggestive of encephalaopathy, no epileptic signs were seen. Spinal fluid was normal. Abdominal ultrasound was normal. Biochemical analysis at that point revealed a significantly increased ammonia levels (enzymatic method with glutamate dehydrogenase - Reagens Roche Catlog Nr. 20766682322 with a Roche Cobas Integra 400 plus machine) of $310 \mu \mathrm{g} / \mathrm{dL}$ (normal < $102 \mu \mathrm{g} / \mathrm{dL}$, see Figure 1), remarkably enough without any other sign of liver disease. Nevertheless, the patient was treated with lactulose enema resulting in ammonia decrease to $216 \mu \mathrm{g} / \mathrm{dL}$ on the fourth day. Neurological status on that time showed a slight improvement with less inconsistent speech and patient answered more appropiately.

On the fifth day, patient was referred to the ICU of our hospital, because of further progressive deterioration of the neurological signs with evolution to comatose state and increasing ammonia levels (1023 $\mu \mathrm{g} / \mathrm{dL})$. Control imaging at admission revealed diffuse cerebral edema. Intracranial pressure monitoring showed significantly increased pressure of $34 \mathrm{mmHg}$. At his time point ammonia was $1453 \mu \mathrm{g} / \mathrm{dL}$.

An inborn error of metabolism of the urea cycle was suspected and chromatography of the plasma amino acids was performed. Awaiting these results, the patient was treated with hemodiafiltration using a high-flux membrane Elisio-21H ${ }^{\circledR}$ (Nipro, Zaventem, Belgium) during 8 hours 45minutes. In addition, ammonia was scavenged by administration of Ammonul \& (Ucyclyd Pharma, Scottsdale, USA), a combination of sodium phenylacetate and sodium benzoate, which creates an alternative pathway to eliminate nitrogen precursors. In total $18.7 \mathrm{~g}$ Ammonul was 
administered in accordance with the dose suggested. These two interventions resulted in a swift decrease of ammonia to $152 \mu \mathrm{g} / \mathrm{dL}$ on sixth day (see Figure 1). A rebound hyperammonemia $(1453 \mu \mathrm{g} / \mathrm{dL})$ occurred 4 hours after dialysis was stopped, which was treated with a new dialysis session. During this session there was an evolution to brain death due to refractory and worsening cerebral edema. All therapy was discontinued and patient died on the seventh day.

Postmortem analysis of the chromatography of the plasma amino acids demonstrated increased levels of glutamine

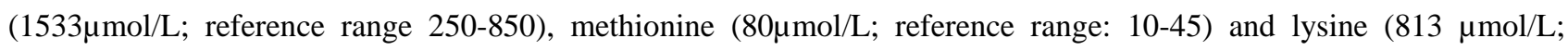
reference range: 50-280). In the urine massively increased concentrations of orotic acid were found, suggestive of ornithine transcarbamylase (EC 2.1.3.3) (OTC) deficiency (MIM \#311250). The diagnosis of OTC was confirmed genetically: c.622G>A (p.Ala208Thr) in the OTC gene in a hemizygous state. This is a known pathogenic mutation, that was described previously in a dutch pedigree with late-onset OTC deficiency ${ }^{[2]}$. Mutation analysis in his relatives revealed that his brother, his daughter and his grandson all carry the pathogenic mutation. At present they are all asymptomatic but remain in follow up to prevent future hyperammonemic crises.

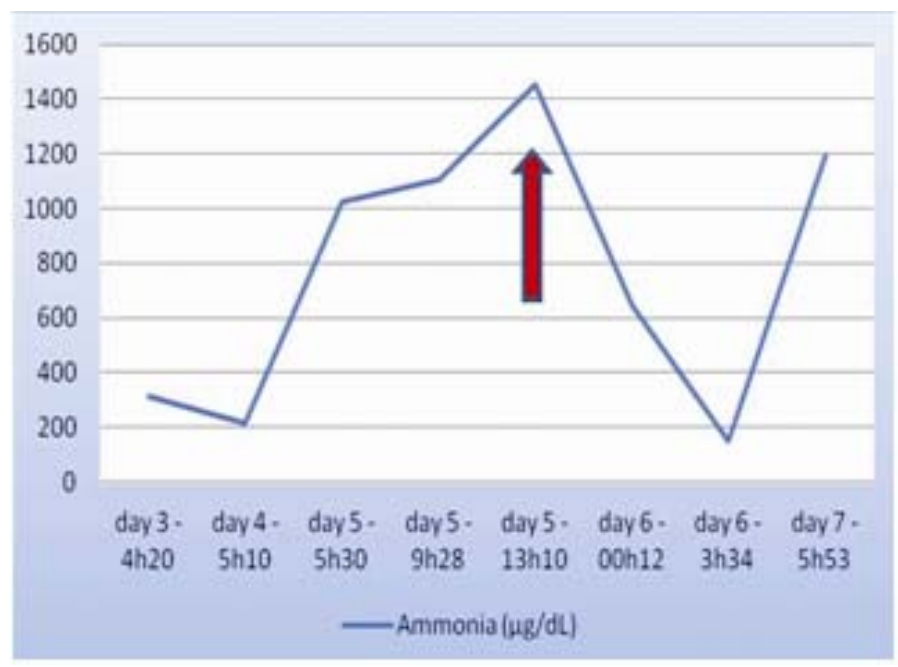

Figure 1. Evolution of ammonia levels after admission. Red arrow indicates start of treatment with dialysis and Ammonul, a preparation of sodium benzoate and sodium phenylacetate. A rapid drop of ammonia levels is observed.

\section{Discussion}

An atypical presentation, mostly due to a mutation causing a partial enzyme inactivity, has been described which is much more difficult to diagnose as symptoms are nonspecific (nausea, sleeping disorders, psychiatric symptoms, vomiting) and occur at older age ${ }^{[3-6]}$. As is illustrated by our case, valuable time can be lost in such cases due to these atypical presenting symptoms and care should be taken to perform an extended biochemical evaluation including ammonia level determination. If this level is found to be elevated in the absence of overt liver disease and if there is a normal anion gap, this should trigger the differential diagnosis of $\mathrm{UCD}^{[7]}$. An increased anion gap indicates the presence of other inborn errors of metabolism (i.e. organic acidaemia, fatty acid oxidation defects, ...) or liver failure.

In majority of the patients with an atypical presentation it is difficult to obtain a clear history. In the absence of a full history (i.e. nutritional status), the threshold has to be kept low to determine an ammonium plasma level in patient with an atypical psychiatric presentation.

Both drugs (Sulpiride and Prazepam) are not known to interact with the pathogenesis of hyperammonemia. It is however well known that Valproate (anti-epileptic drug) can do this. This is why this was not administrated during the period of his 
epileptic seizures. These drugs can however also give psychiatric symptoms (i.e. psychoses, hallucinations, ...) especially in elderly. Since these medications were started 2 months prior to the deterioration and that the patient deteriorated further even after withdrawing these medications, it seems less likely that they are the main reason of this psychotic episode. It seems more likely that the depressive period prior to the psychotic episode had an influence on his nutrition or protein balance. We believe that this has provoked the psychotic episode and the further deterioration.

In most cases plasma amino acid profiles are readily available as are urine orotic acid concentrations to confirm the diagnosis at short notice, some weeks before genetic confirmation. In any case, in doubt swift and adequate treatment is necessary to prevent irreversible brain damage or refractory cerebral edema.

No definite data are available as to which treatment regimen is optimal for the treatment of hyperammonemic crises; however Häberle et al. have suggested some treatment regimens (grade C-D evidence) depending on the ammonia levels ${ }^{[7]}$. The first treatment option is the use of a combination therapy sodium phenylacetate and sodium benzoate, creating an alternative pathway to eliminate nitrogen precursors ${ }^{[8]}$. This can result in a significant and rapid decrease in ammonia levels, hindering evolution to possibly lethal complications like cerebral edema. In the literature, the supplementation of arginine is also recommended since it is an essential amino acid made through the ureum cycle. Due to the enzymatic defect in OTC deficiency, arginine is not produced stimulating further proteinolysis and ammonia accumulation.

If ammonia levels however are too elevated and overt clinical life-threatening symptoms are present, as in our case, hemodialysis treatment should be performed to quickly eliminate the ammonia excess. It has been demonstrated that ammonia elimination by hemodialysis is superior to peritoneal dialysis ${ }^{[9]}$. In hemodialysis this removal is bloodflow, dialysate flow and dialyser surface dependent. If these parameters are maximized, it has been demonstrated that more than $80 \%$ of the present ammonia can be eliminated. The use of a high flux membrane with a large convective flow might even enhance this process ${ }^{[10]}$. Care should be taken to the rebound phenomenon, shortly after dialysis stop. Dialysis does not only lower the concentration of ammonia, but it also lowers the concentration of the administrated sodium phenylacetate and sodium benzoate. Epuration treatment should be tapered slowly while monitoring ammonia levels.

\section{Conclusion}

Our case report illustrates that even at advanced age inborn errors of metabolism may become clinically apparent. It is essential to keep thinking about these rare causes when making a differential diagnosis, as detecting them is pivotal both for the patients' treatment and for the further screening and early detection in his family.

UCDs are a specific subtype of inborn errors of metabolism presenting with non-specific symptoms at young age including gastro-intestinal and neurologic manifestations (i.e. typical presentation). At advanced age, UCDs are more difficult to diagnose because of attenuated phenotypes of which neuropsychiatric symptoms may be the only clinical signs of a more systemic illness. Walterfang et al. listed the inborn errors of metabolism which may present neuropsychiatric symptoms ${ }^{[11]}$.

The association of neuropsychiatric symptoms or encephalopathy of unknown origin and hyperammonemia should lead, even in the absence of liver and kidney disease, to a high level of suspicion of UCDs to ensure a timely diagnosis and life-saving treatment. An inborn error of metabolism, even in male patients, can present at an advanced age. Especially attenuated phenotypes are probably under-ascertained.

\section{References}

[1] Brusilow SW, Maestri NE. Urea cycle disorders: diagnosis, pathophysiology, and therapy. Adv Pediatr. 1996; 43: 127-170. 
[2] Ausems M, Bakker E, Berger R, et al. Asymptomatic and late-onset ornithine transcarbamylase deficiency caused by a A208T mutation: Clinical, biochemical and DNA analyses in a four-generation family. Am. J. Med. Genet. 1997; 68: 236-39. http://dx.doi.org/10.1002/(SICI)1096-8628(19970120)68:2<236::AID-AJMG23>3.0.CO;2-U

[3] Ben-Ari Z, Dalal A, Morry A, et al. Adult-onset ornithine transcarbamylase (OTC) deficiency unmasked by the Atkins' diet. J Hepat. 2010; 52: 292-295. PMid:20031247 http://dx.doi.org/10.1016/j.jhep.2009.11.014

[4] Choi D, Lee K, Shin Y, et al. Hyperammonemia in a patient with late-onset ornithine carbamoyltransferase deficiency. J Korean Med Sci. 2012; 27: 556-559. PMid:22563224 http://dx.doi.org/10.3346/jkms.2012.27.5.556

[5] Panlaqui O, Tran K, Johns A, et al. Acute hyperammonemic encephalopathy in adult onset ornithine transcarbamylase deficiency. Intensive Care Med. 2008; 34(10): 1922-24. PMid:18651132 http://dx.doi.org/10.1007/s00134-008-1217-2

[6] Houston B, Reiss K, Merlo Ch. Healthy but comatose. Am J med. 2011; 124(4): 303-4. PMid:21435419 http://dx.doi.org/10.1016/j.amjmed.2010.12.002

[7] Häberle J, Boddaert N, Burlina A, et al. Suggested guideles for the diagnosis and management of urea cycle disorders. Orphanet J Rare Dis. 2012; 7: 32. PMid:22642880 http://dx.doi.org/10.1186/1750-1172-7-32

[8] Walker V. Ammonia toxicity and its prevention in inherited defects of the urea cycle. Diabetes Obes Metab. 2009; 11: 823-35. PMid:19531057 http://dx.doi.org/10.1111/j.1463-1326.2009.01054.x

[9] Arbeiter A, Kranz B, Wingen A. Continuous venovenous hemodialysis (CVVHD) and continuous peritoneal dialysis (CPD) in the acute management of 21 children with inborn errors of metabolism. Nephrol Dial Transplant. 2010; 25(4): 1257-65. PMid:19934086 http://dx.doi.org/10.1093/ndt/gfp595

[10] Corboba J, Blei A, Mujais S. Determinants of ammonia clearance by hemodialysis. Artif Organs. 1996; 20(7): 800-3. http://dx.doi.org/10.1111/j.1525-1594.1996.tb04544.x

[11] Walterfang M, Bonnot O, Moccilin R, et al. The neuropsychiatry of inborn errors of metabolism. JIMD. 2013; 36(4): 687-702. 